\title{
The Dominant Role of the Chemical Potential for Driving Currents in Oceans and Air
}

\author{
Albrecht Elsner \\ Am Mühlbach 14, D-85748 Garching, Germany \\ Email: alimeli.elsner@gmail.com
}

Received January 2014

\section{Abstract}

Applying the thermodynamic zeros of the entropy $S$ and internal energy $U$ of the gas mass $M$ in the volume $V$ yields the numerically unique relation between these quantities, thus allowing calculation of the chemical potential in the gas fields of temperature $T$ and pressure $p$, viz. $M \mu=U-S T+V p$. A difference in chemical potential provides a force for freely moving matter flow. Since $\mu$ is intrinsically a negative function, decreasing as the temperature increases, natural flow processes are initiated by high $\mu$ values in cold regions directed to low $\mu$ values in warm regions. Modeling the Gulf Stream circulation, calculations show that the force deduced from the chemical potential for certain temperature differences in water outweighs all other forces acting on this current. The chemical potential provides the force not only for flow of matter but also for its changes of phase and for chemical reactions, and thus the chemical potential plays the dominant role in modern theoretical oceanography and meteorology. As an example of a gigantic water flow, the oceanic circulation of the Gulf Stream is investigated. The chemical potential is here introduced for identifying the sources of the forces driving the Gulf Stream. There are two main energy supplies of the Atlantic warm water current: 1) the vertically upwards directed temperature-dependent chemical potential difference reduced by the gravitational hydrostatic energy in the equatorial region, which generates forces that press cold deep seawater from the Atlantic into the Gulf of Mexico, from where the then warm water current finds its pole wards flowing lane at the surface of the North Atlantic, and 2) the vertically downwards directed chemical potential difference enlarged by the gravitational energy in the polar region, which generates forces that continue to transport the then cold surface water to seawater at great depths, from where deep seawater flows back to the Atlantic, thus closing the water circulation in the North Atlantic basin. In general, momentum fluxes in oceans are caused by different seawater conditions at different oceanic locations. The cold water reservoirs at the poles and the warm surface water reservoirs at the equator are the sources of oceanic circulations. Similarly, a cold low-pressure area is the origin of extensive motions of rainy clouds.

\section{Keywords}

Chemical-Potential Temperature Function, Thermo-Mechanical Pressure, Gravitational Pressure, Gulf Stream, Oceanography, Meteorology 


\section{Introduction}

What driving mechanisms are the basis of currents in the atmosphere and currents in oceans? The answers of a meteorologist and an oceanographer concur: On our planet freely moving matter is essentially governed by the forces of gravitation, rotation, friction and, especially in oceans, horizontal wind stress and thermohaline convection. As the densities of air and water greatly differ, atmospheric currents move much faster than oeanic currents. Besides forces, it is ultimately the conservation of mass, energy, momentum, angular momentum and conditions prescribed by continental boundaries that determine the course of currents. The pertinent hydrodynamic equations (Oertel Jr., 2002) are completed with thermodynamic equations (Apel, 1987), which inter-relate parameters such as density, temperature, pressure, enthalpy, heat capacity, compressibility, conductivity etc. In the case of seawater salinity also plays a role; here the thermodynamic relations are compiled in a so-called International Equation of State for Seawater (EOS) (Apel, 1987; Businger, 1992). Evaluation of the Geophysical Fluid Dynamics equations is done by means of powerful computer systems.

Results of these calculations are published in the media. The forecasting in weather reports on the temporal development of air temperature, air pressure, wind intensity, wind direction, cloud formation and precipitation is impressive because it readily predicts the actual weather situation accurately. There are also films of cyclones, whose development can be explained (see Section 4). Impressive are likewise aerial photos of sea currents, e.g. the Gulf Stream. The explanation that the cause of the currents is globally the transfer of horizontal wind momentum to the ocean and thus represents the most energetic component of the oceanic circulation (Apel, 1987) raises, however, doubts. Can it be that it is just winds close to the air-sea interface, whose strength and direction are continually subjected to change, that drive the narrow Gulf Stream band, $100 \mathrm{~km}$ wide and 7000 $\mathrm{km}$ long in the $6000 \mathrm{~km}$ wide North Atlantic, as sole prominent current? Furthermore, a wind-driven wave on the ocean surface forces water masses to move up and down but does not transport them as a current in the direction of the wave propagation. Nevertheless, oceaneographic science holds the view that wind exerts a tangential force on the sea surface that induces a water current with a vertical so-called Ekman spiral. Calculations of the Ekman solution are compared with observations, and differences are specified, viz. a shift of the current by calculated $45^{\circ}$ to the wind direction instead of measured $20^{\circ}$ and a spiral depth of $15 \mathrm{~cm}$ instead of a mean depth of the Gulf Stream of 500 m (Oertel Jr., 2002; Apel, 1987; von der Heydt, 2011). Wind shear is ruled out as explanation for the cold deep-sea currents, which originate at the poles. On the other hand, a physical explanation for their existence is lacking.

The cause of sea currents is obviously a freqently recurring question (von der Heydt, 2011). One must therefore go back to the essential fundamental equations, viz. 1) the momentum equation with the driving force as the sum of the gravitational force, Coriolis force, a frictional force of deceleration, wind stress (Oertel Jr., 2002; Apel, 1987) and possibly a further, thermo-mechanical force of acceleration (see Sections 2 and 3) and 2) the thermodynamic relations formulated by UNESCO (Apel, 1987). This makes study of a thermodynamic textbook (e.g. Callen, 1960) inevitable.

Thermodynamics treats the physical theory of the reversible equilibrium states of a macroscopic system as well as its irreversible processes (Callen, 1960). The theory emphasizes two facts: 1) A complete state description of a thermodynamic system by the system parameters: temperature $T$, pressure $p$ and chemical potential $\mu$ and 2) the fact that a chemical-potential gradient generates a flow of matter. The circumstance that this knowledge has not featured in the disciplines, oceanography and meteorology, is solely due to the fact that the chemical potential cannot be calculated as long as the thermodynamic value of the internal energy of the system as an absolute value is unknown. Numerical determination of the thermodynamic reference values of entropy $S$ and internal energy $U$, which are assigned to the system mass $M$ in volume $V$, has fundamentally changed the situation (Elsner, 2012). The absolute values of $M, V, S, U, T$ and $p$ make it possible to calculate the energy value of the chemical potential:

$$
M \mu=U-S T+V p
$$

It is customary to calculate with mass-specific quantities, i.e. with $(M \mu) / M=\mu, U / M=u, S / M=s$, $V / M=v=1 / \rho$, where $\rho$ is the density. For water as an example, Figure 1 presents $\mu$ as a function of $T$. The general and essential properties of the chemical potential between absolute zero and the critical temperature can be seen: $\mu$ is a continuous, negative, concavely curved, decreasing function of $T$. It thus holds that every matter of freely moving particles flows from high $\mu$ values, i.e. from cold regions to low $\mu$ values, i.e. to 


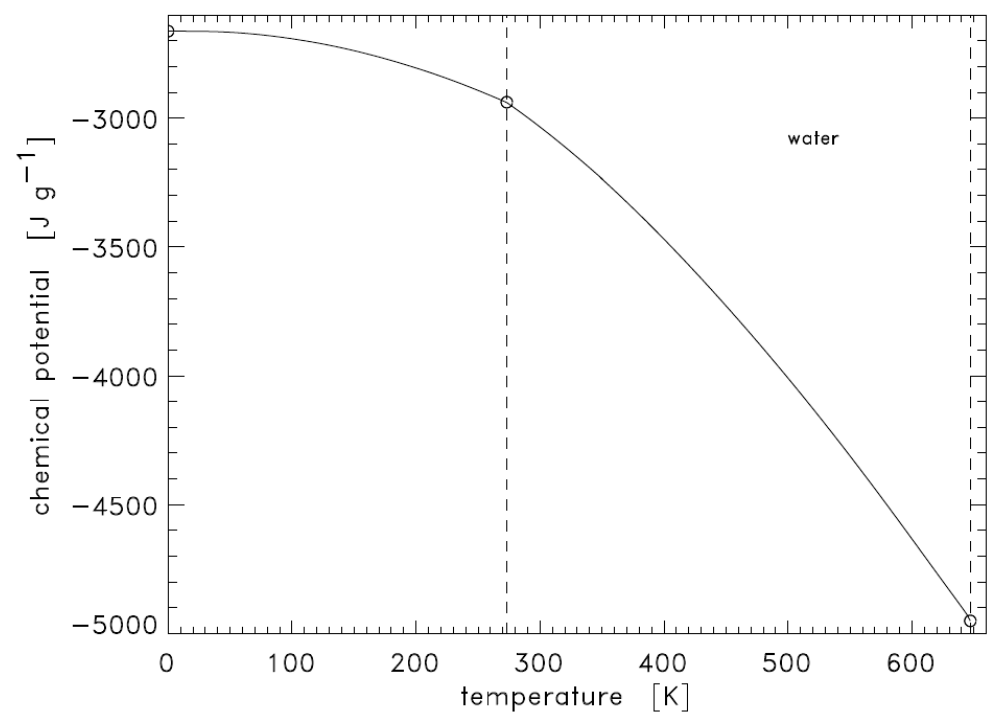

Figure 1. Chemical potential of water between the absolute zero and critical points. At the triple point, $\mu$ is continuous but the derivatives are discontinuous where $d \mu / d T_{\text {liquid }}<d \mu / d T_{\text {ice }}<0$.

warm regions.

That a particle flow is due to interaction forces within the fluid is shown by the fact that within the fluid a gradient of energy $M \mu$ exerts a force on every fluid particle and acts as a force driving a current, this being similar to the converse, where in a flowing fluid frictional forces occur between the particles and reduce the kinetic energy of the fluid. The interactions between the particles are also responsible for the variation of the particle motion when the fluid is subjected to an external mechanical pressure, which changes the volume-pressure energy. The situation is different when the particle motion is subjected to gravitational forces and Coriolis forces; here external forces act on every particle mass $m$, independently of its interaction with other particles. The stated internally generated and externally imposed forces $F$, which set in motion the fluid, i.e. the mass $M=\sum m$ in the volume $V$, are treated as body forces $f=F / M$ (unit: dyne/g) or as volume forces $\bar{f}=F / V$ (unit: dyne/ $\mathrm{cm}^{3}$ ). It holds that $\bar{f}=f / v=f \rho$.

The oceanic water circulation of the Gulf Stream represents a continuous system of gigantic extent. It is truly impressive how pregnantly this was described by American naval officer M. F. Maury in his book entitled "The Physical Geography of the Sea” (1855): There is a river in the ocean. In the severest droughts it never fails, and in the mightiest floods it never overflows. Its banks and its bottom are of cold water, while its current is of warm. The Gulf of Mexico is its fountain, and its mouth is in the Arctic Seas. It is the Gulf Stream (Apel, 1987).

In Sections 5 and 6, Maury's assertions are thermodynamically confirmed by investigating the sources of forces that drive and confine the warm water. In order to handle the problem of driving forces in different oceanic regions, a highly simplified oceanic model is considered to be a basin composed of six different homogeneous volumes $V_{n}$ of densities $M_{n} / V_{n}=\rho_{n}$ under the local conditions $\left[T_{n}, p_{n}, \mu_{n}\right]$. It is intended to compare the two main energy sources, viz. the hydrostatic and thermo-mechanical pressures in each volume $V_{n}$. Typical Gulf Stream data allow calculation and comparison of gravitational and thermo-mechanical energies at different geographic locations (see Section 6).

\section{Flow of Matter Due to a Chemical Potential Gradient}

Thermodynamics says that in an inhomogeneous fluid there may also be, besides gravitational and mechanical forces, thermo-mechanical forces which generate flow of matter within the fluid from regions of high chemical potential to those of low potential.

Let us consider the mass flow between two volumes $V_{1}$ and $V_{2}$ in which water is retained under the con- 
ditions, $\left\{\rho\left(T_{1}\right),\left[T_{1}, p\left(T_{1}\right), \mu\left(T_{1}\right)\right]\right\}$ and $\left\{\rho\left(T_{2}\right),\left[T_{2}, p\left(T_{2}\right)+\rho\left(T_{2}\right) g H, \mu\left(T_{2}\right)\right]\right\}$, where $\rho$ is the local density (Apel, 1987), $T$ the temperature, $p$ the vapor pressure, $\mu$ the chemical potential, $g$ the gravitational acceleration, $H$ the vertical distance between $V_{1}$ and $V_{2}$, and $p\left(T_{2}\right)+\rho\left(T_{2}\right) g H$ the hydrostatic pressure in $V_{2}$. The flow depends not only on these external thermal conditions, but also on the local surroundings and the viscosity of water. If the viscosity can be neglected, i.e. water flows without any friction loss, the flow is governed by the balanced external conditions, viz. the differences between chemical-potential and hydrostatic energies, $\left[\left(\mu_{1}-\mu_{2}\right)-\left(V_{1} \rho\left(T_{1}\right)-V_{2} \rho\left(T_{2}\right) g H\right]\right.$, where the positive sign determines the flow direction, say, from volume 1 to volume 2. Before calculating $\left(\mu_{1}-\mu_{2}\right)$, one has to consider the well-known Gibbs relation (with $v=1 / \rho$ ) (Callen, 1960),

$$
d \mu d T=v d p d T-s<0
$$

which describes the temperature gradient of the chemical potential, i.e. $d \mu / d T$ as the difference between the function $v d p / d T$ and the entropy $s$. Figure 2 shows the temperature dependence of these functions for water. Note that $d \mu / d T$ does not depend on the water phase, since it holds that $[v d p / d T-s]_{\text {fluid }}=[v d p / d T-s]_{\text {condensate }}=[v d p / d T-s]_{\text {vapor }}$. Integration of Equation (2) yields (with $\left.v_{1}=v_{2}=v\right)$

$$
\left(\mu_{1}-\mu_{2}\right)=\int_{T_{2}}^{T_{1}} d \mu d T d T=v\left(p_{1}-p_{2}\right)+\int_{T_{1}}^{T_{2}} s(T) d T>0 \quad \text { for } \quad T_{1}<T_{2}
$$

Equation (3) once again states that $\mu$ decreases as $T$ increases.

As regards pressures, Equation (2) is multiplied by $\rho$, yielding $d p / d T-\rho s<0$. One then obtains the pressure relation

$$
P_{\mu}=\int_{T_{1}}^{T_{2}} \rho(-d \mu d T) d T=-\left(p_{2}-p_{1}\right)+\int_{T_{1}}^{T_{2}} \rho s d T>0 \quad \text { for } \quad T_{1}<T_{2}
$$

The thermo-mechanical pressure $\int_{T_{1}}^{T_{2}}(\rho s) d T$ is also termed fountain pressure as discussed in the context of the helium flow in HeII (Allen \&Jones, 1938). The vapor pressure difference $\left(p_{2}-p_{1}\right)=\int_{T_{1}}^{T_{2}}(d p / d T) d T$ is

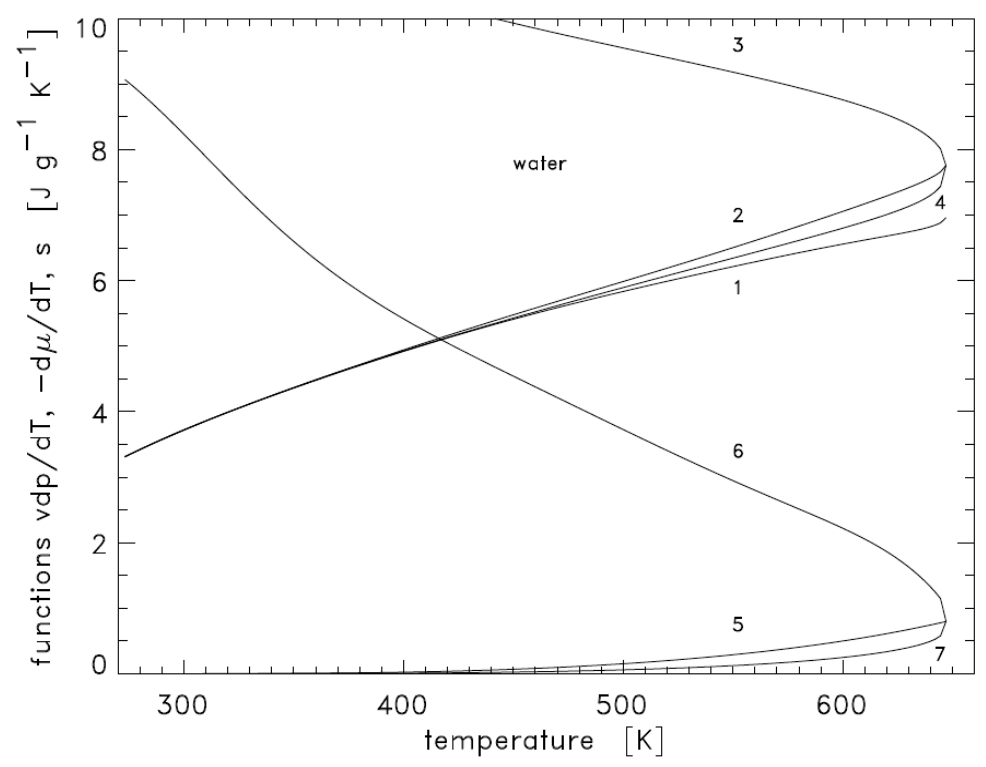

Figure 2. Functions of water between the triple and critical points. 1: $-d \mu / d T=s-v d p / d T>0,2: s, 3: s_{\mathrm{v}}, 4: s_{\mathrm{l}}, 5: \quad v d p / d T, 6: v_{\mathrm{v}} d p / d T$, and 7: $v_{1} d p / d T$. Subscript v: vapor phase, subscript l: condensate phase (liquid water, ice). 
directed counter to the thermo-mechanical pressure. The imbalance (4) gives the principal source of flow dynamics within an inhomogeneous fluid. Relation $P_{\mu}>0$ thus imposes the condition for matter flow from volume 1 with $T_{1}$ to volume 2 with $T_{2}$ if $T_{1}<T_{2}$. In the case of the Gulf Stream the lateral pressures $P_{\mu}$ are directed from its cold surrounding to its warm center and confine it, including the large warm and cold core rings that exist for weeks after their detachment from the meandering main stream (Apel, 1987). Vertical pressures at the sources and sinks of the Gulf Stream maintain the current and its prescribed direction.

\section{Equation of Momentum}

According to statistical mechanics and in agreement with Newton, the change with respect to time $t$ of the quantity, motion of mass $M$, moving with velocity $u$, viz. the momentum $M u$, is caused by the force $F$ imposed on the moving mass $M$ :

$$
d d t(M u)=d M d t u+M d u d t=F
$$

In oceanography and meteorology it is convenient to consider the flow of the volume $V$ containing $M$, and, consequently, from Equation (5) one obtains $d(M / V) d t u+M V d u d t=F V, d \rho d t u+\rho d u d t=\bar{f}$, and $d \ln (\rho) d t u+d u d t=\bar{f} \rho$ or

$$
d \ln (\rho) d t u+d u d t=f
$$

Important forces $f_{i}$ come from five sources: gravity, Coriolis effects, mechanical pressures, chemical-potential-dependent thermo-mechanical pressures and friction, where it holds that $f=\sum f_{i}$. Whereas the sea water density in oceans is nearly constant, the density of air in the atmosphere rapidly varies. Thus the term $d \ln (\rho) / d t \cdot u$ in the hydrodynamic Equation (6) can be dropped when treating seawater, but must be regarded when treating air currents. The relevant chemical-potential force $f_{\mu}$ along the path $d s$ of changes in energy $\mu(T)$ can be expressed as follows:

$$
f_{\mu}=-d \mu d s=d T d s(-d \mu d T)=\text { gradient } T \cdot(s-1 \rho d p d T)
$$

The direction of $f_{\mu}$ is the same as that of gradient $T$, since $(s-1 / \rho d p / d T)>0$. Relations (7) express $f_{\mu}$ as a function of entropy $s$, density $\rho$ and the differences in $T$ and $p$.

\section{Driving Forces of Air}

The atmospheric flow of air is governed by the exchange of heat and moisture between air masses and the rapid variation in momentum and torque acting on them.

Examples of atmospheric flow of matter in nature are: A hurricane develops vortical transport of water vapor from a warm seawater surface (more than $25 \mathrm{C}$ ) to the troposphere (altitudes between 14 and $19 \mathrm{~km}$ ) and forms there around an inner core (of up to $30 \mathrm{~km}$ in diameter) walls of rainy cumulus clouds of considerable height (of up to $1 \mathrm{~km}$ ) and extent (of up to $1000 \mathrm{~km}$ in diameter). The hurricane vortex can exist 2 - 4 days and vanishes after leaving its energy source, the ocean, setting free all water masses on land (Allen \& Jones, 1938). The development of a hurricane can be explained as follows: By thermal buoyancy warm water vapor from the ocean surface is driven up to cold air, where it condenses to rainy cloud parcels. These then cold parcels are subjected to thermo-mechanical and Coriolis forces and vortically transported around the warmer inner core of a beginning cyclone in the troposphere. Subsequently, the inner core remains warm due to permanently rising water vapor and release of heat of vaporization, so that the condition for thermo-mechanical forces towards the vortex center is sustained to balance in part the centrifugal force. The increasing vapor masses expand the core in width and height by condensation and in lower regions by frictional effects and thus the clouds are kept in expanding circular paths of motion. The cumulative water and air masses rotating around the core, steadily driven by Coriolis forces, travel with increasing velocity over sea and land until they lose water supply and mass. The motion of cold air falling at night to warmer air layers below, there condensing to fog and dew, and rising in the daytime to air layers above which it gets warmer and warmer by sun irradiation. Cold draughts through canyons and, at home, through gaps in windows and doors at equal atmospheric pressures outside and inside. Currents in inhomogeneous, electrically not neutral fluids in the form of flashes of lightning between clouds and 
of ions and electrons in plasma discharge devices, the cause here being not the chemical potential difference but the electrochemical potential difference.

\section{Sources of Driving Forces of the Gulf Stream}

In the following, the origin of oceanic flows is explained. To be specific, the given external conditions which lead to the current of the Gulf Stream in the Atlantic are discussed here.

For this purpose, consider the water volume $V$ comprising six subvolumes $V_{n}$, where each $V_{n}$ is characterized by the homogeneous state parameters $\rho_{n}$ and $\left(T_{n}, p_{n}, \mu_{n}\right)$, giving the different external conditions in $V$. Thus, the inhomogeneities of the density, temperature, pressure and chemical potential in $V$ are described by the local parameters $\rho_{n}$ and $\left(T_{n}, p_{n}, \mu_{n}\right)$. The actual density $\rho_{n}$ depends nonlinearly on the local values of temperature, pressure and salinity, and is calculated by the UNESCO formula, ranging roughly from 1.03 $\left[\mathrm{g} \cdot \mathrm{cm}^{-3}\right]$ to $1.05\left[\mathrm{~g} \cdot \mathrm{cm}^{-3}\right]$. At atmospheric pressure, seawater freezes at $-1.8[\mathrm{C}]$ and has its maximum density $\rho_{\max }$ (Apel, 1987).

The Gulf Stream can be highly simplified as a current cycle in a seawater basin comprising six subvolumes $V_{1}, \ldots, V_{6}$. It should be noted that each volume is characterized by water-specific properties and not by fixed dimensions. The boundaries of the warm-water bed of the Gulf Stream, viz: the bottom and banks of the river (Maury), are physically determined by the higher chemical potential of the colder surrounding Atlantic water (at depths below 500 [m] and between the continental shelves). This, on the one hand, determines the north-easterly direction of the current and minimizes the flow cross-section and, on the other, leads to continuous cooling and spatial expansion of the Gulf Stream along its course, as a result of lateral inundation of cold water. The following water properties in the 6 individual subvolumes are considered:

$V_{1}$ : seawater in the polar region around Iceland, which is cooled at the surface by ice and cold winds to freezing temperature $T_{1}=-1.8[\mathrm{C}]$ and has atmospheric pressure $p_{1}$ and maximum density $\rho_{1}=\rho_{\max }$, $\rho_{\max }=1.05\left[\mathrm{~g} \cdot \mathrm{cm}^{-3}\right]$ and chemical potential $\mu_{1} \cdot V_{2}$ : deep seawater below $V_{1}$ at a depth $H \geq 2000$ [m], with $T_{2}=4[\mathrm{C}], \rho_{2}<\rho_{\max }, \rho_{2}=1.04\left[\mathrm{~g} \cdot \mathrm{cm}^{-3}\right], p_{2}=p_{1}+g \rho_{2} H, \mu_{2}<\mu_{1} . V_{3}$ : deep seawater between Iceland and the North American shelf with $\rho_{3}=\rho_{2}, T_{3}=T_{2}, p_{3}=p_{2}, \mu_{3}=\mu_{2} . V_{4}$ : deep seawater on the North American shelf with $\rho_{4}=\rho_{3}, T_{4}=T_{3}, p_{4}=p_{3}, \mu_{4}=\mu_{3} . V_{5}$ : water above $V_{4}$, where cold deep seawater displaces warm equatorial water from the Gulf of Mexico, with $\rho_{5}<\rho_{4}, \rho_{5}=1.03\left[\mathrm{~g} \cdot \mathrm{cm}^{-3}\right]$, $T_{5}=25$ [C], $p_{5}=p_{1}, \mu_{5}<\mu_{4} . V_{6}$ : water at the surface between the Gulf of Mexico and Arctic Seas (Gulf Stream between $V_{5}$ and $V_{1}$ ), with $\rho_{6}=\rho_{5}, T_{6}=T_{5}, p_{6}=p_{1}, \mu_{6}=\mu_{5}$.

In $V_{3}$ and $V_{6}$, water streams in opposite directions as required for continuity of flow; the external conditions cause no driving forces either between $V_{2}$ and $V_{3}, V_{3}$ and $V_{4}$, or $V_{5}$ and $V_{6}$. The driving forces of the current between $V_{1}$ and $V_{2}$ derive from the chemical potential difference $\left(\mu_{1}-\mu_{2}\right)$ and hydrostatic energy of the weight of the water column $H$. Both the thermo-mechanical and the hydrostatic pressures act in the same direction from the surface to the sea bottom. Further driving forces of the current between $V_{4}$ and $V_{5}$ are caused by the chemical potential difference $\left(\mu_{4}-\mu_{5}\right)$ and hydrostatic energy; here $\mu_{4}[4 \mathrm{C}]$ is more positive than $\mu_{5}$ [25 C], i.e. the relevant thermo-mechanical pressure is counterdirected to the hydrostatic pressure. Since the thermo-mechanical pressure outweighs the hydrostatic pressure, the force is directed from the sea bottom to the surface.

In the water flow direction the sum of the two hydrostatic pressure drops approximately vanishes, $-\left(\rho_{1}-\rho_{2}\right) g H+\left(\rho_{4}-\rho_{5}\right) g H=\left(2 \rho_{2}-\left(\rho_{\max }+\rho_{5}\right)\right) g H \approx(2.08-2.08) g H=0 \quad$ [MPa]. The thermo-mechanical pressures in $\left(V_{1}, V_{2}\right)$ and $\left(V_{4}, V_{5}\right)$ are obtained from the expression $\int_{T_{1}}^{T_{2}}(\rho s) d T+\int_{T_{4}}^{T_{5}}(\rho s) d T \approx \int_{T_{1}}^{T_{5}}(\rho s) d T$. The total flow-driving pressure is thus given by

$$
P_{\text {Gulf Stream }}=\int_{T_{\mathrm{ps}}}^{T_{\mathrm{ds}}}(\rho s) d T+\int_{T_{\mathrm{ds}}}^{T_{\mathrm{es}}}(\rho s) d T=\int_{T_{\mathrm{ps}}}^{T_{\mathrm{es}}}(\rho s) d T
$$

where ps = polar seawater, ds = deep seawater, es = equatorial seawater.

Equation (8) can be evaluated as follows: Without distinguishing between seawater and freshwater, the estimates yield with $T_{\mathrm{ps}}=0[\mathrm{C}], T_{\mathrm{ds}}=4[\mathrm{C}]$ and $T_{\mathrm{es}}=25[\mathrm{C}]$ the total thermo-mechanical pressure value 88 [MPa] (see Figure 3). This shows that the Gulf Stream is essentially driven thermo-mechanically. The main 


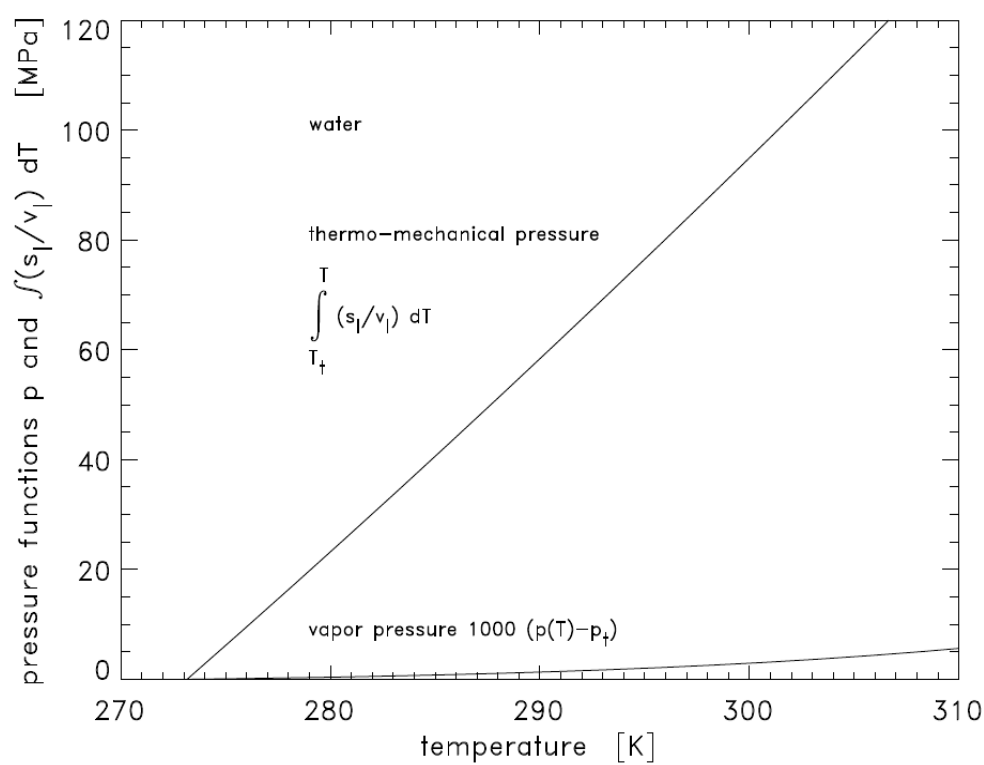

Figure 3. The functions $\int_{T_{t}}^{T}\left(s_{1} / v_{1}\right) d T$ and $1000 \cdot\left(p(T)-p\left(T_{t}\right)\right)$ of liquid water above the triple-point temperature $T_{t}$. Note that $\left(s_{1} / v_{1}\right)=\left(\rho_{1} s_{1}\right)$. Numerical values at $298[\mathrm{~K}]$ : thermo-mechanical pressure $=88[\mathrm{MPa}]$, vapor pressure $1000 \cdot\left(p(T)-p\left(T_{t}\right)\right)=3.2[\mathrm{MPa}$. The hydrostatic pressure at a depth of $3300[\mathrm{~m}]$ is 33 [MPa].

pressure contribution of $77[\mathrm{MPa}]$ is made by the chemical potential difference ensuing from the confluence of cold deep seawater and warm surface water from the Gulf of Mexico, viz. $\int_{T_{\mathrm{ds}}}^{T_{\mathrm{es}}}(\rho s) d T$. The contribution of 11 [MPa] of surface water in the Arctic Seas sinking to the region of deep seawater results from $\int_{T_{\mathrm{ps}}}^{T_{\mathrm{ds}}}(\rho \mathrm{s}) d T$.

Evaluation of chemical potential differences according to Equation (3) and Figure 2 yields $\left(\mu_{273}-\mu_{277}\right)=(-2938+2949)=11[\mathrm{~J} / \mathrm{g}]$ and $\left(\mu_{277}-\mu_{298}\right)=(-2949+3026)=77[\mathrm{~J} / \mathrm{g}]$. Multiplying these values by the density $1\left[\mathrm{~g} / \mathrm{cm}^{3}\right]$ also gives the above-mentioned pressures $11[\mathrm{MPa}]$ and 77 [MPa]. The hydrostatic pressure at a depth of $3300[\mathrm{~m}]$ is 33 [MPa].

\section{Gulf Stream Data}

After comparison of the balanced hydrostatic and thermo-mechanical pressures one should now consider the Gulf Stream data (Apel, 1987; von der Heydt, 2011)) measured in the four subregions of the circulation, $V_{1} \rightarrow V_{2}, V_{2} \rightarrow V_{4}, V_{4} \rightarrow V_{5}, V_{5} \rightarrow V_{1}$. The discussion here takes a very simplified form.

The Gulf Stream (in $V_{6}$ between $V_{5}$ and $V_{1}$ ) is $L=2.5 \cdot 10^{6}$ [m] long, $W=10^{5}$ [m] wide, and

$D=8 \cdot 10^{2}[\mathrm{~m}]$ deep, i.e. it has a water volume $V_{6}=L W D=2 \cdot 10^{14}\left[\mathrm{~m}^{3}\right]$. Of this $\dot{V}_{6}=8 \cdot 10^{7}\left[\mathrm{~m}^{3} \cdot \mathrm{s}^{-1}\right]$ flows through the Atlantic at a velocity of $u=1\left[\mathrm{~m} \mathrm{~s}^{-1}\right]$. The warm equatorial seawater thus needs $t_{u}=L / u=V_{6} / \dot{V}_{6}=2.5 \cdot 10^{6}[\mathrm{~s}]$ or about 1 month to reach the vicinity of the North Pole. When fully cooled from $25[\mathrm{C}]$ to $0[\mathrm{C}]$ by lateral incursion of colder seawater and by winds, it transfers in this time to the surroundings, viz. North Atlantic water and air, a heat of

$Q_{\text {heat to surroundings }}=V_{6} \rho c \Delta T=Q_{u}=2 \cdot 10^{14}\left[\mathrm{~m}^{3}\right] \cdot 10^{6}\left[\mathrm{~g} \mathrm{~m}^{-3}\right] \cdot 4\left[\mathrm{~J} \mathrm{~g}^{-1} \mathrm{C}^{-1}\right] \cdot 25[\mathrm{C}]=2 \cdot 10^{22} \quad$ [J]. This is equivalent to a power of $Q_{u} / t_{u}=8 \cdot 10^{15}[\mathrm{~W}]$.

The circulation of the Gulf Stream mass $M=\rho V_{6}=2 \cdot 10^{20}$ [g] includes a kinetic energy $E_{u}=M 2 \cdot u^{2}=10^{17}[\mathrm{~J}]$. If the water in the polar region sinks with a velocity $u_{v}=0.1\left[\mathrm{~m} \mathrm{~s}^{-1}\right]$ to the 
deep-water region, it reaches a depth $H=3 \cdot 10^{3} \quad[\mathrm{~m}]$ after $t_{v}=H / u_{v}=3 \cdot 10^{4} \quad$ [s]. The energy thus released is $E_{g}=g M H=6 \cdot 10^{21}[\mathrm{~J}]$ and this is again expended when deep water rises along the North American coast. Accordingly, the energy balance of the circulation can dispense with the contribution of the compensating potential energies, viz. $g M H+g M(-H)=0$. As the water sinks and rises, a power $E_{g} / t_{v}=g M u_{v}=2 \cdot 10^{17}$ $[\mathrm{W}]$ is being permanently converted. The flow of water from the polar region to the Gulf of Mexico is subject to turbulences and hence friction losses, which are set equal to the kinetic energy $E_{v}=M 2 \cdot u_{v}^{2}=10^{15} \quad[\mathrm{~J}]$.

Sustaining the circulation, i.e. the sum of the kinetic energies $E_{u}$ and $E_{v}$, requires an energy source, this being present owing to differences of the chemical potential due to different water temperatures in different oceanic regions. In addition, there exists yet another energy source which acts as a wind-driven oceanic circulation at a shallow depth below the water surface in the north-easterly flow direction; its value is negligible in comparison with the chemical-potential energy. The total circulation energy $E_{\text {chemical potential }}+E_{\text {wind shear }}$ can be estimated. According to Figure 2, the exchange of polar water of $0[\mathrm{C}]$ and deep seawater of $4[\mathrm{C}]$ yields the chemical potential energy $M\left(\mu_{273 \mathrm{~K}}-\mu_{277 \mathrm{~K}}\right)=2 \cdot 10^{20}[\mathrm{~g}] \cdot 11\left[\mathrm{~J} \cdot \mathrm{g}^{-1}\right]=2.2 \cdot 10^{21} \quad[\mathrm{~J}]$; and the exchange of deep seawater and equatorial water of $25[\mathrm{C}]$ yields the energy $M\left(\mu_{277 \mathrm{~K}}-\mu_{298 \mathrm{~K}}\right)=2 \cdot 10^{20}[\mathrm{~g}] \cdot 77\left[\mathrm{~J} \cdot \mathrm{g}^{-1}\right]=1.5 \cdot 10^{22}$ $[\mathrm{J}]$; this makes a total of about $E_{\text {chemical potential }}=1.7 \cdot 10^{22}[\mathrm{~J}]$, and adding the wind-driven energy $E_{\text {wind shear }}$ gives an estimated value $2 \cdot 10^{22}[\mathrm{~J}]$. This energy is equal to the heat $Q_{\text {heat to surroundings }}=2 \cdot 10^{22} \quad[\mathrm{~J}]$, taken from the Gulf of Mexico and transferred to the environment. The circulation energy is available as long as 4 [C] deep seawater in the Gulf of Mexico displaces an equal mass of 25 [C] warm water and this is cooled on the way to the polar region to $0[\mathrm{C}]$ surface water through heat transfer.

\section{Results and Discussion}

Meteorologists and oceanographers are concerned with flows of air in the troposphere and water in oceans, and with exchange of mass, momentum and energy at the interface of air and water. Studies of the circulation of oceanic currents concur in concluding that the currents are driven by surface flux, mainly wind shear at the ocean surface and thermohaline convection in deep water ((Apel, 1987; von der Heydt, 2011). The basis of this theory and conclusions from it yield the equation of state (UNESCO 1981 formula) for the thermodynamic conditions and the Navier-Stokes differential equations for the dynamic processes. Despite all efforts to clarify what drives an ocean current, no satisfactory answer has hitherto been forthcoming.

The chemical potential is a decisive quantity in the equation of state and must therefore be taken into account when describing flow of matter in inhomogeneous fluids. It has a higher value at low temperature than at high temperature, which can immediately be deduced from Gibbs's relation $\rho d \mu / d T=-\rho s+d p / d T<0$. In the case of free-particle transport it thus follows that matter always flows from a region of high chemical potential and low temperature to one of low chemical potential and high temperature. This is how the well-known fountain effect in helium physics is explained as well as the work done by an engine in the ocean (Ocean Thermal Energy Conversion, OTEC) (Baretta-Bekker, Duursma, \& Kuipers, 1998). It is important in oceanography and meteorology to know that it is, besides Coriolis and centrifugal forces, mainly the existence of thermo- mechanical and gravitational forces exerted on the fluid that effects the motion of sea currents and air.

In this paper it is shown that Maury's description of the Gulf Stream is correct: by the flow of cold deep seawater into the Gulf of Mexico, the warm water there is channeled by the presence of land masses in the north-easterly direction. Along its entire course the warm water is then kept together, as the Gulf Stream, by incursion of surrounding, colder ocean water, being continuously cooled and thus being subjected to confinement loss. Furthermore, the current at the free surface also transfers heat to the colder air. It sinks close to the 60th degree of latitude in vertical channels of $10^{3}$ [m] diameter as $0[\mathrm{C}]$ surface water with a high velocity of up to $0.1\left[\mathrm{~m} \cdot \mathrm{s}^{-1}\right]$ to the deep seawater of the permanent 4 [C] (von der Heydt, 2011).

The sources needed to sustain the North Atlantic circulation, while giving off heat to the surroundings from rapidly flowing warm water of narrow extent at the surface and driving backwards slowly flowing, cold, deep seawater of wide extent at the bottom, are the vertical thermo-mechanical and gravitational energy potentials in the Gulf of Mexico and the polar region. Since the total water current is steady, the forces in the equatorial and 
polar regions must be balanced. When comparing the strengths of the upwards and downwards driving forces, one has to set the corresponding expressions for the upwards and downwards pressures equal, viz. the relevant sums of the thermo-mechanical and hydrostatic pressures. With equal values of the upwards and downwards hydrostatic pressures $\rho g H$ being taken for granted, this gives $\left(\int_{4 C}^{25 C}(\rho s) d T-\rho g H\right)-\left(\int_{0 C}^{4 C}(\rho s) d T+\rho g H\right)=0$ or $(77-\rho g H)-(11+\rho g H)=0$ (in [MPa] units). The model described allows the averaged pressures for driving the Gulf Stream to be estimated as $44[\mathrm{MPa}]$ and the depth at which the water flows back as $3.3 \cdot 10^{3}$ [m]. This should be compared with the contribution resulting from the momentum transfer of wind at the water surface and the efficiency of the Ekman layer and its depth, which varies from some 10 [cm] to 100 [m] (Apel, 1987).

The analysis of the large-scale oceanic circulation resembles that of an electric circuit. The two Kirchhoff rules apply: both the sum of the voltage drops around a closed loop and the sum of the currents into a node vanish. A sufficiently large set of measured data (such as the World Ocean Circulation Experiment, WOCE) should thus make it possible to identify the sources and currents in the global network of oceans and the troposphere. The local and temporal variations of the energy sources and currents are very much more pronounced in the troposphere than in the oceans, where, in turn, they are the weaker, the deeper the sea. It will transpire that the pictures of the oceanic circulation, viz. the wind fields over the ocean combined with Coriolis effects and, in deeper parts of the ocean, the thermohaline processes, which are simply assumed to be generated in proportion to the differences in water temperature and salinity, are not tenable and should be replaced essentially by the pictures of chemical-potential and hydrostatic energy differences. The energy for maintaining the existence of oceanic circulation is supplied by the sun. About half of the incident solar radiation is absorbed by oceans, where it heats the upper levels of the sea. This amount contributes to circulation of water and evaporation across the sea-air interface (Apel, 1987).

\section{Acknowledgements}

The author would like to thank Institut für Plasmaphysik (IPP), Garching, for providing computing facilities. He is also grateful to A. M. Nicol for the English translation.

\section{References}

Allen, J. F., \& Jones, H. (1938). New Phenomena Connected with Heat Flow in He-II. Nature, 141, 243-244. http://dx.doi.org/10.1038/141243a0

Apel, J. R. (1987). Principles of Ocean Physics. Academic Press, Chapters 1.2, 2, 3, 4, 6.

Baretta-Bekker, Duursma, \& Kuipers (1998). Encyclopedia of Marine Sciences. Springer, 110.

Brockhaus, V. (2012). Wissenswelten: Geografie, Natur, Klima. Mohn Media, 596-600.

Businger, J. A. (1992). Ocean-Atmosphere Dynamics, Equations (1)-(7). In R. A. Meyers, Ed., Encyclopedia of Physical Science and Technology (Vol. 11). Academic Press.

Callen, H.B. (1960). Thermodynamics. John Wiley \& Sons, Chapters 3.2, 3.3, 6.4, 16.

Elsner, A. (2012). Applied Thermodynamics of the Real Gas with Respect to the Thermodynamic Zeros of the Entropy and Internal Energy. Physica B: Physics of Condensed Matter, 407, 1055-1067. http://dx.doi.org/10.1016/j.physb.2011.12.118

Oertel Jr., H. (2002). Prandtl-Führer durch die Strömungslehre. Vieweg, 11. Auflage, Chapters 4.1.1, 5.1, 5.2.1, 12.2.4, 12.4.1.

von der Heydt, A. (2011). Die Physik der Ozeanströme. Physik Journal, 10, 23-29. 